\title{
Multiple Model Approach to Uptake and Distribution of Halothane*: The Use of an Analog Computer
}

\author{
Aart Zwart, N. Ty Smith, $\uparrow$ And Jan E. W. Beneken \\ Institute of Medical Physics, T.N.O. Department: Cardiovascular Physics, \\ Utrecht, The Netherlands
}

Received October 4, 1971

\begin{abstract}
This paper describes a multiple analog computer model of the uptake and distribution of the anesthetic agent halothane. The model consists of two interdependent loops, one representing the blood circulation and another representing the halothane transport. Cardiac output and regional conductances are influenced in relation to the concentration of halothane in some relevant compartment of the model. Computer generated curves are given to show the response of alveolar concentration, arterial pressure and cerebral blood flow to step and pulse changes in inspired concentration. For evaluation of the model, other variables than alveolar concentration, such as, arterial pressure and blood flow may often be morc uscful. The present model will be used to develop an automatic control system for the administration of anesthesia.
\end{abstract}

\section{INTRODUCTION}

Since the beginning of the century, models have been used to describe the uptake and distribution of inhalation anesthetic agents in the body as shown by Kety (I) and Papper (2). All but one (3) have been linear models. In a linear model, cardiac output and its distribution to the different body compartments are constant during the uptake of the anesthetic. In reality, cardiac output and ventilation are dependent on the level of anesthesia. Recently, a considerable amount of quantitative data about regional blood flow has become available under different steady state levels of halothane anesthesia (4).

We, therefore, incorporated these data into a nonlinear analog computer model, which can make interdependent changes in cardiac output and its distribution as a function of concentration of halothane in one or two of the three most important compartments i.e., brain, myocardium and arterial blood.

* Supported in part by Ayerst Laboratories and Hoechst Holland N.V.

$\dagger$ Recipient of NIH Research Career Development Award 1K3-GM-31, 757-05. 


\section{METHODS}

The concept of the model is the same as Mapleson (5) describes and is shown in Fig. 1.

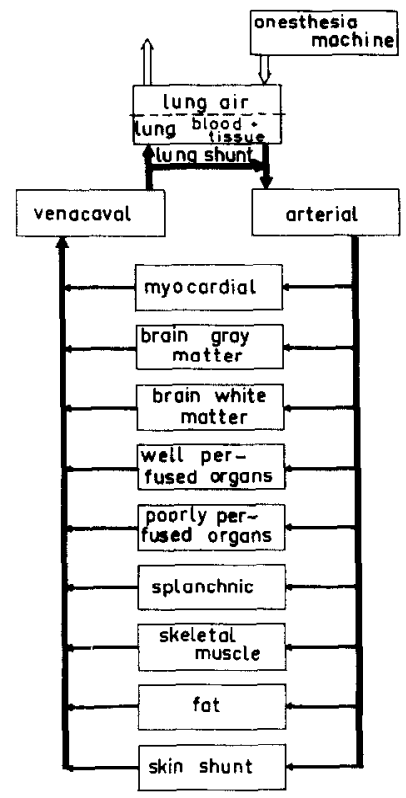

FIG. 1. A generalized scheme of the compartmental division of the model. Arterial blood is partitioned over nine compartments and collected in the vena cava. Venous blood goes to the lung or through a lung shunt to the arterial compartment.

Our basic assumptions are as follows:

1. The model can be realized with a finite number of compartments.

2. Ventilation and blood flow can be described as nonpulsatile phenomena, since equilibration times are large compared to the cardiac or respiratory cycles.

3. Transport times are negligible, for a similar reason.

4. Ventilation is kept constant, because it is usually artificial.

5. There is no other way of exchange between different compartments than transport by the blood.

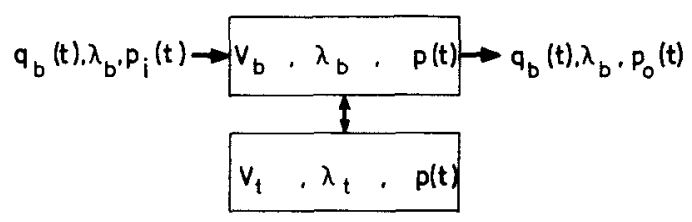

FIG. 2. Setup of a standard compartment which consists of tissue and blood which have the same concentration. 
6. Equilibration within a compartment is instantaneous.

7. The inhalation agent is not metabolized.

8. There is no adaptation to halothane.

With these assumptions, we can describe an individual compartment (Fig. 2) with the following equations:

$$
\begin{aligned}
& p(t)=p_{0}(t), \\
& \alpha(t)=\alpha(0)+\int_{0}^{t} \lambda_{b} q_{b}(t)\left[p_{i}(t)-p_{0}(t)\right] d t, \\
& \alpha(t)=p(t)\left(\lambda_{b} V_{b}+\lambda_{t} V_{t}\right) .
\end{aligned}
$$

Equations (1)-(3) give

where

$$
p(t)=p(0)+\frac{1}{\lambda_{b} V_{b}+\lambda_{t} V_{t}} \int \lambda_{b} q_{b}(t)\left[p_{i}(t)-p(t)\right] d t,
$$

$\alpha=$ total amount of agent in the compartment,

$\lambda_{b}=$ solubility coefficient for blood,

$\lambda_{t}=$ solubility coefficient for tissue,

$p=$ partial pressure of agent in the compartment,

$p_{i}=$ partial pressure of agent in the blood entering the compartment,

$p_{0}=$ partial pressure of agent in the blood leaving the compartment,

$q_{b}=$ volume flow of blood,

$V_{b}=$ blood volume in equilibrium with the tissue of the compartment,

$V_{t}=$ tissue volume of the compartment.

Three compartments are described a little differently:

1. The lungs. Along with the blood flow there is the airflow entering at inspired concentration, leaving at expired concentration. We also have to consider, besides the equilibrated blood and tissue volume, the air volume in the lungs. The equation becomes

$$
p(t)=p(0)+\frac{1}{\lambda_{b} V_{b}+\lambda_{t} V_{t}+V_{a}} \int_{0}^{t}\left\{\lambda_{b} q_{b}(t)\left[p_{V i}(t)-p(t)\right]+q_{v}(t)\left[p_{i l}(t)-p(t)\right]\right\} d t,
$$

where

$V_{a}=$ functional residual capacity +0.5 tidal volume,

$p_{V i}=$ partial pressure of halothane in venous blood,

$p_{i i}=$ partial pressure of halothane in inspired air,

$q_{v}(t)=$ ventilation of the lung corrected for dead space (alveolar ventilation). 
2. The arterial compartment. Two blood flows enter the compartment: one flow of blood equilibrated in the lungs, the other one of blood with venous concentration. Venous blood entering the arterial compartment can have several sources, such as perfusion of a lung section without ventilation, or a by-pass of the lung (right to left shunt). We call both phenomena lung shunt.

3. The venous compartment. Several flows enter the compartments each with its own partial pressure of halothane.

For the arterial and venous compartments, the equation becomes

$$
p(t)=p(0)+\frac{1}{\lambda_{b} V_{b}+\lambda_{t} V_{t}} \int_{0}^{t} \lambda\left[\sum q(t) p_{i}(t)-\operatorname{CO} p(t)\right] d t
$$

where

$$
C O=\text { total cardiac output, }
$$

$\sum q(t) p_{i}(t)=$ summation over all flows entering the compartment.

\section{The Circulation Loop}

Parallel to this section of the model is a circuit (Fig. 3), dealing with cardiac output and its distribution to the different compartments. An extensive description of the use of such parallel circuits was given by Beneken (6):

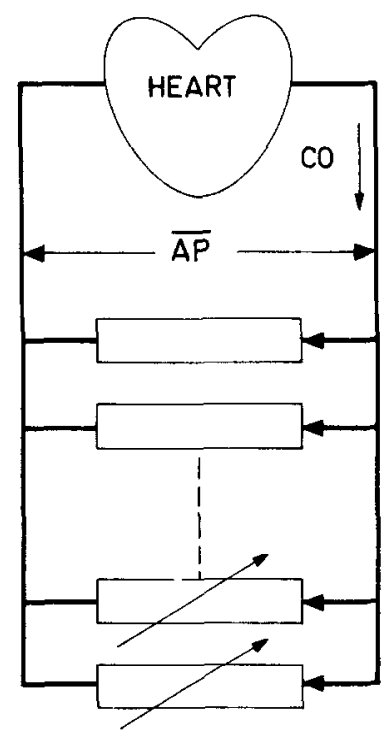

FIG. 3. Sctup of the circulation circuit. Cardiac output is divided among nine parallel compartments which have fixed conductance (open boxes) or variable conductance (boxes with arrows). Mean arterial pressure is computed as pressure drops over these parallel compartments. 
In our parallel circuit, we consider the heart to deliver a flow according to the function

$$
\begin{aligned}
& \qquad O=C O(0)(1+a p), \\
& C O= \text { total cardiac output, } \\
& C O(0)= \text { total cardiac output prior to anesthesia, } \\
& a= \text { constant, } \\
& p= \text { partial pressure of halothane in that compartment which, dependent } \\
& \text { on the hypothesis, determines cardiac output. We used three different } \\
& \text { compartments, arterial blood concentration, brain (gray matter) con- } \\
& \text { centration (nervous influence) or myocardial concentration. }
\end{aligned}
$$

We describe the conductance of the $j$ th compartment as

$$
G_{j}=G_{j}(0)\left(1+b_{j} p\right)
$$

$G_{j}=$ conductance of the $j$-th compartment,

$G_{j}(0)=$ conductance of the $j$-th compartment prior to anesthesia,

$b_{j}=$ constant of the $j$-th compartment,

$p=$ partial pressure of halothane in that compartment which, dependent on the hypothesis, determines conductance.

In this set up, mean arterial pressure becomes

$$
\overline{A P}=C O / \sum_{j} G_{j},
$$

and the blood flow to the $j$-th compartment

$$
q_{b j}=\overline{A P} \times G_{j} .
$$

\section{RESULTS}

To show the use of our multiple model, we present some curves obtained with this model. To compare linear models with multiple models, the model was also used as a linear model which is realized if the flow is kept constant. In the multiple model we tried different modes of control. With mode of control, we mean the mechanism involved to change circulation variables as indicated by the Eqs. (5) and (6). Figure 4 illustrates the change in alveolar concentration of halothane after a step-change from 0 to $2 \%$ in inspired concentration. The curves generated from the linear models give a reasonable approximation of the curves drawn with a multiple model. We see that the concentration curves for different hypotheses about modes of control fall very close together. In the first few minutes, the curves generated by the multiple model are close to the alveolar concentration curve from the linear model with awake bloodflows. Later on, they approximate the curve from the linear model with blood flow at a $2 \%$ equilibrium level. Figure 5 shows how sensitive alveolar concentration curves are to changes in lung shunt. Figures 6 and 7 show that there are variables such as mean arterial pressure and cerebral blood flow, which give considerably 


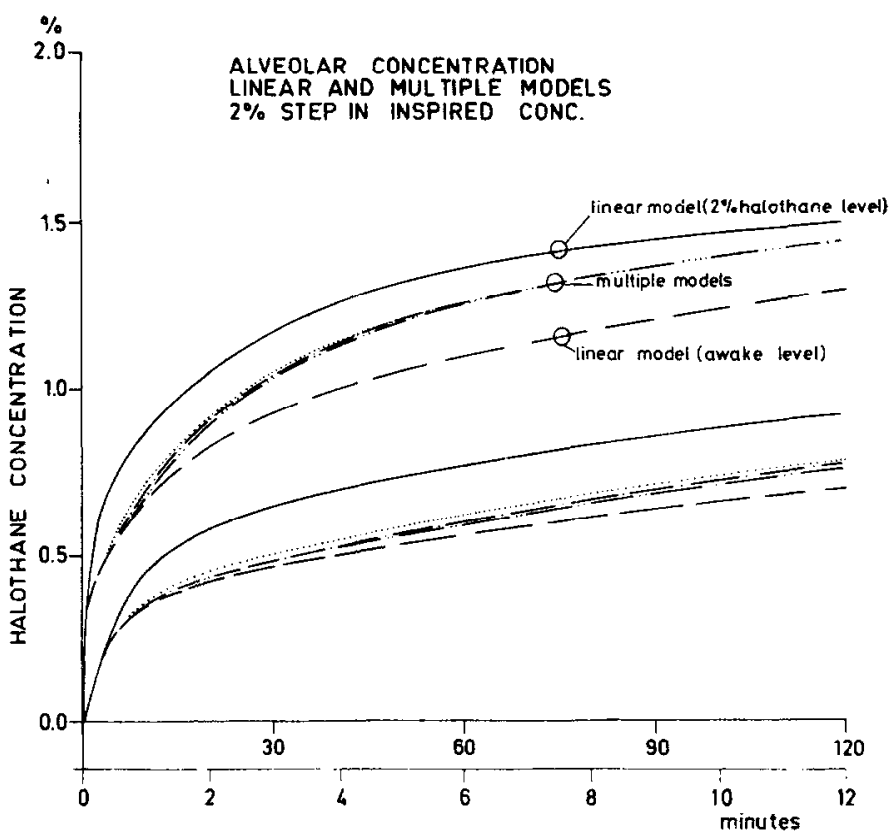

FIG. 4. Response of alveolar concentration to a step change from 0 to $2 \%$ in inspired halothane concentration. Five curves are shown in the upper group-two generated with a linear model, three with a multiple model. The lower group of curves shows the first $12 \mathrm{~min}$ of the upper group. The solid line is drawn with a linear model with the flow data at $2 \%$ equilibrium level of halothane; the dashed line with flow data at awake level. The other curves are drawn with multiple models. For all three curves, conductances were controlled by arterial concentration. Only the control of cardiac output was changed. ..... CO controlled by arterial concentration, $-\cdot \cdot-\cdot-. \mathrm{CO}$ controlled by concentration in brain gray matter, -..... CO controlled by myocardial concentration.

different curves for different hypotheses about modes of control after a pulse change in inspired concentration. With such a pulse change, the inspired concentration is changed to a new level for $1 \mathrm{~min}$ after which we return to the original inspired concentration.

\section{Discussion}

Many objections can be raised against the way we derive pressure from cardiac output and its distribution, Eq. (7). The actual mechanism in the body might work the other way round and many mechanisms are neglected, for instance, baroreccptors or venous tone. Our first aim was to investigate if a multiple model might be useful in the field of uptake and distribution of halothane and the chosen functions $(5-8)$ were suitable for that purpose and furthermore easy to realize. We learned from the model that alveolar concentration is not sensitive to different modes of control of the circulation, but is sensitive to changes in lung shunt. This makes 


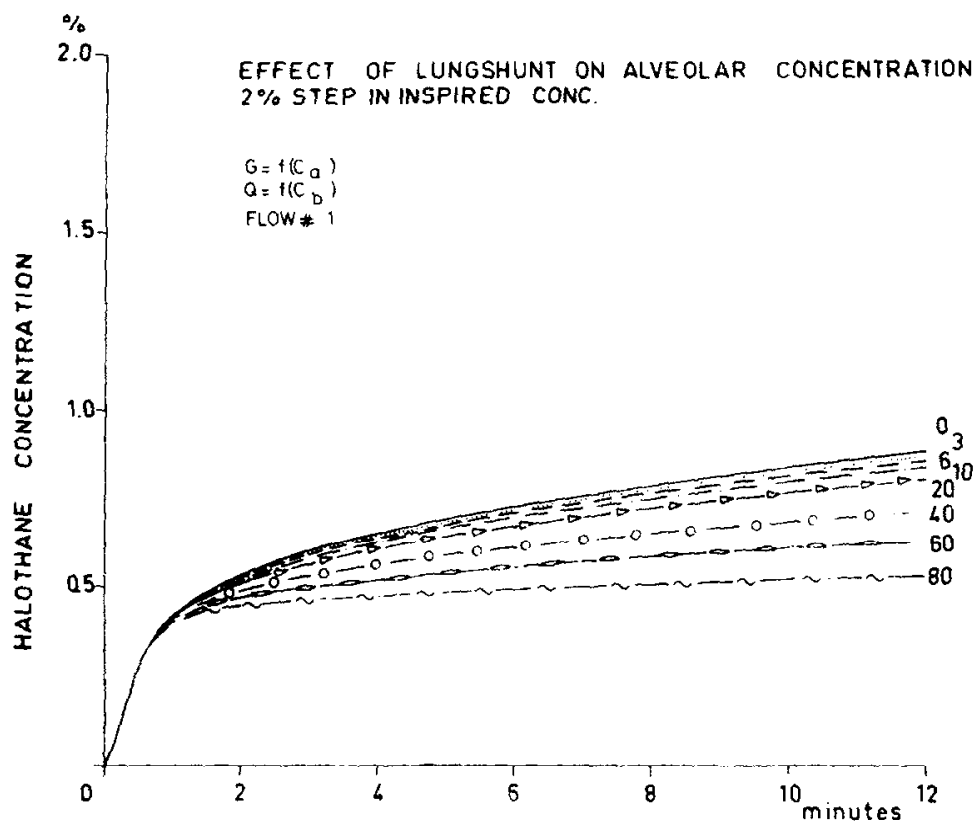

Fic. 5. Response of alveolar concentration to a step change from 0 to $2 \%$ in inspired concentration with different lung shunts. The numbers in the figure indicate the percentage of lung shunt. Note the differences between these curves compared to the differences in the curves for the multiple models in Fig. 4.

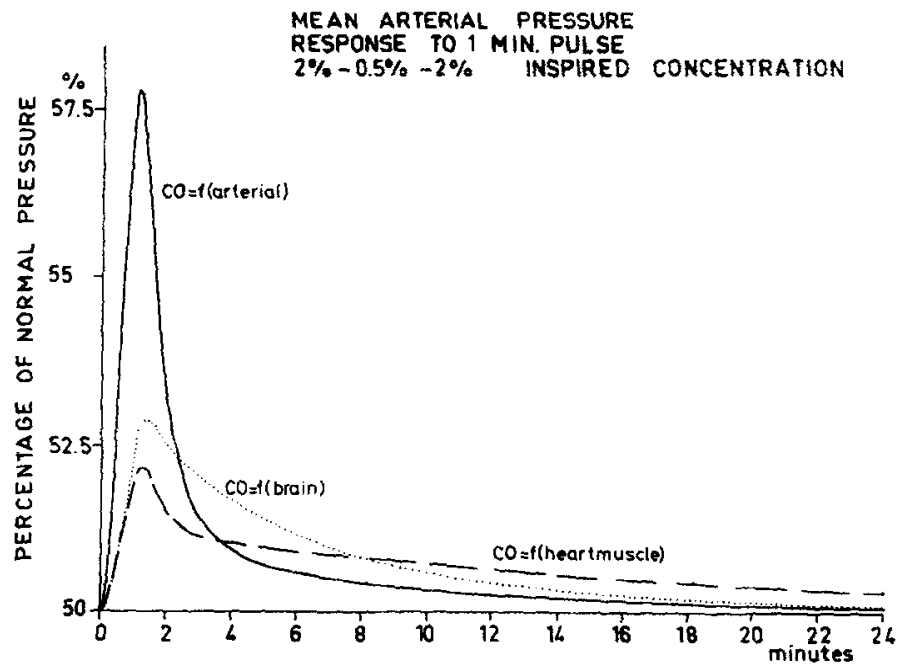

Fig. 6. Response in $\overline{A P}$ on a 1 -min. pulse in inspired halothane concentration from $2.3-0.5-2.3 \%$ Multiple model conductances for all curves controlled by arterial concentration. CO controlled by arterial concentration (solid), concentration in the brain gray matter (dotted) and myocardial concentration (dashed). This means the same modes of control have been used as in the multiple models of Fig. 4. 


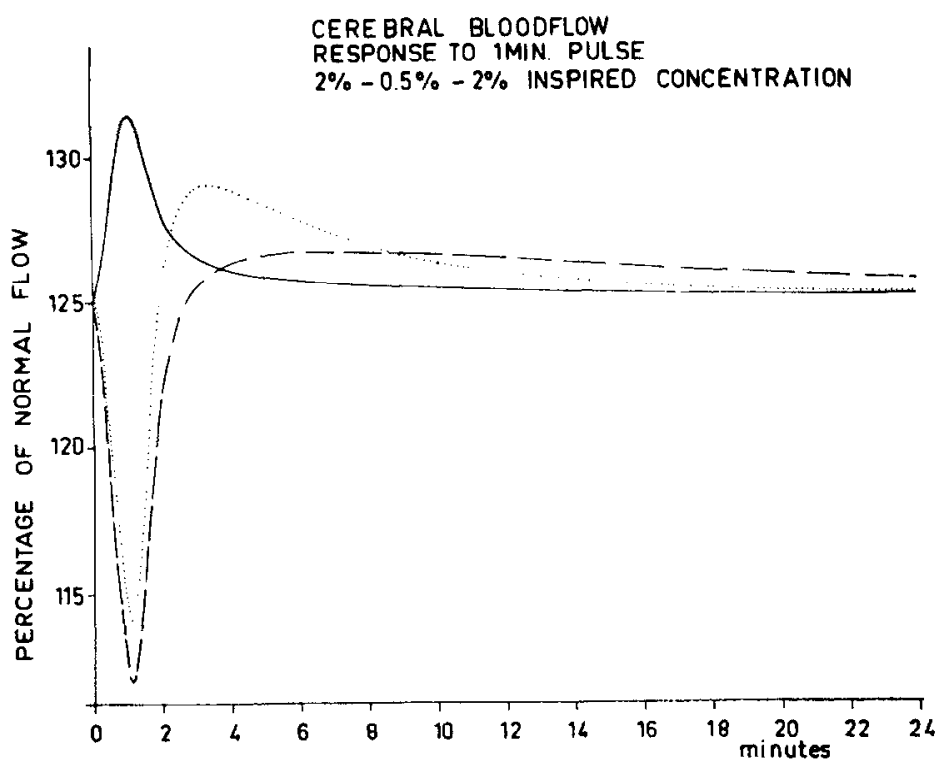

FIg. 7. Response in cerebral blood flow on a 1-min. pulse in inspired halothane concentration from 2.3-0.5-2.3\% of inspired halothane concentration. Modes of control are the same as in Fig. 6 .

experimental distinction of different modes of control with the help of alveolar concentration an illusion. On the other hand, circulation variables like mean arterial pressure and blood flow to different compartments are sensitive to different modes of control. With the help of the circulatory variables, it seems to be possible to evaluate the hypothesis of the control mechanism and improve the functions for $\overline{A P}, C O$ and conductance with the help of animal experiments. By measuring aortic flow and pressure, we can independently evaluate cardiac output functions and changes in total conductance. For evaluating the model with animal experiments, step, pulse and sinusoidal changes in input concentrations will be used. A disadvantage of the sinusoidal method in animal experiments is that it is time consuming. One must use sinewaves of periods ranging from 60 to $2000 \mathrm{sec}$.

With the help of the model we can point out which information we can expect from the different variables and how to minimize the influence of changes in the experimental set up such as lung shunt which is difficult to avoid and to measure.

The present model enables us to test an automatically controlled anesthesia system with multiple inputs such as, e.g., mean arterial pressure, alveolar concentration and E.E.G. (7). Alveolar concentration can be measured as end tidal concentration so that these variables are clinically easily obtainable. With the model, we can study the response time and stability of the automatic control system and which individual circulatory variations are permissible. In this way premature experiments on animals and patients can be avoided. 


\begin{abstract}
APPENDIX
Tables 1-3 give the data which were used for the model. These tables are the same as used by N. Ty Smith in his article, "Interaction Between the Circulatory Effects and the Uptake and Distribution of Halothane", submitted to Anesthesiology in 1971 , where he gives the rationals for our choice. Lung shunt was kept at $3 \%$, if not stated otherwise.
\end{abstract}

TABLE 1

Miscellaneous Data

\begin{tabular}{ll}
\hline \multicolumn{1}{c}{ Parameter } & Value \\
\hline Total ventilation & $61 / \mathrm{min}$ \\
Alveolar ventilation & $41 / \mathrm{min}$ \\
Alveolar tidal volume & $0.30 !$ \\
Physiologic dead space & $0.15 !$ \\
Volume of anesthetic apparatus & 0.661 \\
Lung shunt & $3 \%$ \\
Functional residual capacity & 2,51 \\
\hline
\end{tabular}

TABLE 2

Blood Volumes, Tissue Volumes, and Partition Coefficients FOR A $75 \mathrm{~kg}$ MAN

\begin{tabular}{|c|c|c|c|}
\hline Compartment & $\begin{array}{l}\text { Blood volume } \\
\text { (liters) }\end{array}$ & $\begin{array}{l}\text { Tissue volume } \\
\text { (liters) }\end{array}$ & $\begin{array}{l}\text { Partition } \\
\text { coefficient }\end{array}$ \\
\hline Arterial & 0.96 & -- & 2.3 \\
\hline Brain /gray & 0.371 & 0.75 & 5.4 \\
\hline Brain white & 0.100 & 0.75 & 8.3 \\
\hline Heart & 0.148 & 0.30 & 8.1 \\
\hline Well-perfused organs ${ }^{a}$ & 0.876 & 0.34 & 3.7 \\
\hline Poorly-perfused tissue ${ }^{b}$ & 0.117 & 6.2 & 5.3 \\
\hline Fat $^{c}$ & 0.160 & 12.2 & 138 \\
\hline Splanchnic $^{d}$ & 0.976 & 3.9 & 6.0 \\
\hline Skeletal muscle ${ }^{e}$ & 0.410 & 33 & 8.1 \\
\hline Skin shunt & 0.40 & $\ldots$ & $\ldots$ \\
\hline Vena cava & 0.56 & - & $\ldots$ \\
\hline Lung & 0.372 & 0.6 & 5.3 \\
\hline
\end{tabular}

${ }^{a}$ Well-perfused organs $=$ kidneys, adrenal glands and thyroid gland.

${ }^{b}$ Poorly-perfused tissue $=$ red marrow and nonfatty subcutaneous.

${ }^{c}$ Fat $=$ fat and fatty marrow.

d Splanchnic $=$ organs drained by portal and hepatic circulations.

e Muscle $=$ muscle and skin nutritive. 
TABLE 3

Blood Flows: Awake and at $2 \%$ Halothane

\begin{tabular}{lccc}
\hline \multicolumn{1}{c}{ Compartment } & $\begin{array}{c}\text { Blood flow_awake } \\
(1 / \mathrm{min})\end{array}$ & $\begin{array}{c}\text { Blood flow-2\% Halothane } \\
(1 / \mathrm{min})\end{array}$ & $\begin{array}{c}\text { Percent } \\
\text { change }\end{array}$ \\
\hline Arterial (cardiac output) & 5.800 & 3.480 & -40 \\
Brain gray $_{\text {white }}$ & 0.600 & 0.750 & +25 \\
Heart & 0.150 & 0.188 & +25 \\
Well-perfused organs $^{a}$ & 0.250 & 0.125 & -50 \\
Poorly-perfused tissue $^{b}$ & 1.280 & 0.277 & -78 \\
Fat $^{c}$ & 0.190 & 0.095 & -50 \\
Splanchnic $^{d}$ & 0.200 & 0.100 & -50 \\
Skeletal muscle $^{e}$ & 1.430 & 0.715 & -50 \\
Skin shunt & 1.200 & 0.480 & -60 \\
Vena cava $_{\text {Lung }}$ & 0.500 & 0.750 & +50 \\
& 5.800 & 3.480 & -40 \\
\hline
\end{tabular}

${ }^{a}$ Well-perfused organs = kidneys, adrenal glands, and thyroid gland.

${ }^{b}$ Poorly perfused tissue $=$ red marrow and nonfatty subcutaneous.

${ }^{c}$ Fat $=$ fat and fatty marrow.

${ }^{d}$ Splanchnic $=$ organs drained by portal and hepatic circulation.

${ }^{e}$ Muscle $=$ muscle and skin nutritive.

Figure 8 shows the setup of a standard compartment which generates Eq. (4).

Amplifier $S_{1}$ forms $\left(p_{i}(t)-p(t)\right)$ which is multiplied by $M$ with the factor $q_{b} / q_{b}(0)$. Potentiometer $\mathrm{P}$ represents the factor $\lambda_{b} q_{b}(0) /\left(\lambda_{b} V_{b}+\lambda_{t} V_{t}\right)$ so that integration by $I$ takes place over

$$
\left[p_{i}(t)-p(t)\right]\left(q_{b} / q_{b}(0)\right)\left[\lambda_{b} q_{b}(0) /\left(\lambda_{b} V_{b}+\lambda_{t} V_{t}\right)\right]
$$

and $S_{2}$ is used as inverter.

As input for $M$ in compartments with varying conductance, $q_{b} / q_{b j}(0)$ is used $\overline{A P} / \overline{A P}(0)$ in the compartments with constant conductance and $(1+a p)$ for those compartments receiving total cardiac output.

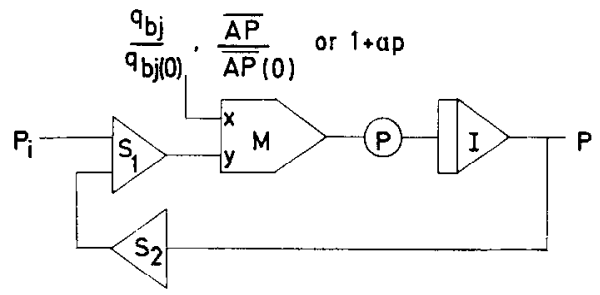

FIG. 8. Machine realization of a standard compartment. For explanation, see text. 
The output $p$ of a compartment is used as $p_{t}$ for the next compartment. Figure 9 shows the setup of the circulation loop. The amplifier $S_{\text {co }}$ generates the slightly altered Eq. (5) as it generates the fraction of $C O$ at awake level $(1+a p)=C O / C O(0)$.

Potentiometer (a) represents the constant $a$ from Eq. (5), $p$ is the input voltage representing the concentration of halothane in a compartment from the uptake and

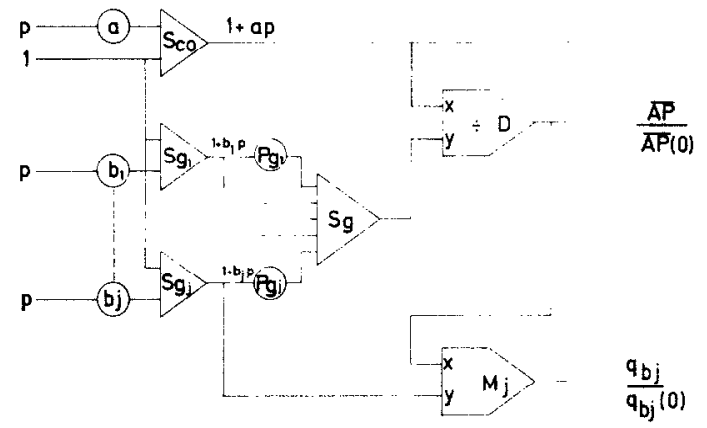

FIG. 9. Machine realization of the circulation loop. For explanation, see text.

distribution loop. 1 is a constant equal to unity. Amplifiers $S$ generate, in an analogous way, Eq. (6) giving $1+b_{j} p=G_{j} / G_{j}(0)$.

The potentiometers $P_{g j}$ represent the fraction of the total conductance for the $j$-th compartment so that $S_{g}$ gives the change in total conductance $\sum\left(G_{j} / G_{j}(0)\right)$, Eq. (7) slightly altered is generated by divider $D$ giving $\overline{A P} / \overline{A P}(0)$, and $E q$. (8) slightly altered by multiplier $M_{j}$ giving $q_{b j} / q_{b j}(0)$.

For this model we use 27 multipliers and 57 amplifiers used as summer or integrator.

\section{ACKNOWLEDGMENTS}

The authors acknowledge the invaluable assistance of J. J. van der Meer, Experimental Laboratory of the Department of Cardiovascular Surgery, University Hospital, Utrecht, The Netherlands. We are also indebted to Mr. J. A. P. Weber and Mr. A. van Dieren from the Institute of Medical Physics, T.N.O. for their technical assistance.

\section{REFERENCES}

1. KeTY, S. A. Pharmacol. Rev. 3, (1 (1951).

2. PAPPER, E. M. AND KITZ, R. J. "Uptake and Distribution of Anesthetic Agents." McGraw-Hill, New York, 1963.

3. Ashman, M. N., Blesser, W. B. and Epstein, R. M. Anesthesiol. 33, 419 (1970).

4. Sмттн, N. TY. AND SMIth, P. C. Circulatory effects of modern inhalation anesthetic agents. In "Modern Inhalation Anesthetic Agents," Heffter's Handbook of Experimental Pharmacology. Springer-Verlag, Berlin, (In press).

5. Mapleson, W. W. J. Appl. Physiol. 18, 197 (1963).

6. Beneken, J. E. W. AND Rideout, V. C., I.E.E.E. Trans. B.M.E. 15, 281-298 (1968).

7. Lopes da Silva, F. H. Smith, N. Ty, ZWART, A., AND Nichols, W. W. Spectral Analysis of the EEG during Halothane "Anesthesia, Input-Output Relations" Electroenceph. clin. Neurophysiol. (In press). 